\title{
Design on Algorithm and Mechanism of Smog Image Sharpening Procession
}

\author{
Zhao $\mathrm{Li}^{1, \mathrm{a}}$ \\ ${ }^{1}$ Shandong Yingcai University, Ji'nan, Shandong, 250104, China \\ azhaolifriend@163.com
}

KeyWords: smog image; sharpening; Retinex algorithm; evaluation

\begin{abstract}
: this paper makes simulation and analysis on several kinds of algorithms such as global, SSR, MSR, MSRCR etc in Retinex algorithm, it summarizes the advantages of MSR algorithm and makes improvement on it, it also puts forward to combine global, part with Retinex algorithm together, meanwhile it makes gain compensation, it further makes comprehensive sharpening procession algorithm after histogram balance. The simulation result indicates that this algorithm can increase the overall contrast ratio of image, improve the confuse problems in details, and better keep color of image. Afterwards, it demonstrates this algorithm has better sharpening effect from subjective and objective aspects.
\end{abstract}

\section{Introduction}

With the quick development in computer technology, people are paying more attention to the visual system of computer and universally applying it into every aspect ${ }^{[1,2]}$ in society. In smog weather, visibility if scene has greatly decreased, which will cause traffic jam, blockage in express way, boat suspension, it is urgent for people to find proper ways to make up for the limitations of peoples eyes in smog weather, on the other hand, computer outdoor monitoring system such as road monitoring etc needs to clearly input image. In smog weather, image has low contrast ratio and bad color fidelity, which has caused serious threat ${ }^{[3,4]}$ on precision of basic intelligent identification, target tracking. Therefore, the sharpening process of degraded image has important realistic meanings, which has obtained attention from more and more scholars. However, computer visual system is very sensitive to weather condition, especially in the smog weather condition with low visibility, which has greatly limited and affected effect display of outdoor computer visual system.

This paper uses improved filtration with multi-scale and minimum to make optimization on transmissivity for the image defogging based on dark channel prior with plenty of matting calculation and high time complication, the improved algorithm through test demonstration enhances the operation speed of algorithm and keeps the defogging effect of image.

\section{Image procession technology and method in smog weather}

Complicated structure and unpredicted change of atmosphere has plenty of effect on light, these unstable disturbance will cause light intensity or color to have changes, so that it will reduce image quality. Through research people find that disturbance of atmosphere on light can be divided into 3 kinds, absorption, reflection and scattering, of which, the important cause on image degradation in smog weather is the scattering effect caused by particle matter in the air. It can be seen that the above-mentioned 2 conditions appear at the same time under the real smog weather condition through incident light reduction model and air light imaging model, the combined effect of these 2 parts cause reduction in image quality. The image taken in the smog weather is the sum of incident light reduction model and air light imaging model, which is indicated as follows:

$$
I(x)=t(x) J(x)+A(1-t(x))
$$

The first item in the right of formula is incident light reduction model, it is direct reduction item and describes the process of target reflection light being reduced, the second item in the right of 
formula is air light imaging model, which reflects the image formed by environmental light and causes fuzzy scene and bias distortion in color. This equation is defined in the RGB color channel, I represents the observed image with smog, $\mathrm{A}$ is the overall air light (it is usually supposed as overall constant, so the overall air light is irrelevant to part position $\mathrm{x}$ ), $\mathrm{J}$ is the radiance of scene, that is the imaging brightness of direct reflection by light scene target, it is the image that needs to be recovered by us, $t(x)$ indicates the medium transmission rate of smog. When air is even with the same quality, it is the scattering coefficient, $d$ is dept of scene. The target of defogging is to recover $J(x), A$ and $t(x)$ from I(x). This is the core idea for scholars to use atmosphere model to realize scene sharpening.

$$
t(x)=e^{-\beta d(x)} \quad(2)
$$

Under the smog weather condition, the image collected by visual system is usually fuzzy with low contract ratio, but it still needs to retain certain character sic information of scene target. Image enhancement is the basic content in image procession, which is to extrude certain characteristic information by designated requirements so that image after enhancement is much more conforms to subjective vision or computer identification system. It is indicated by diagram 1 .

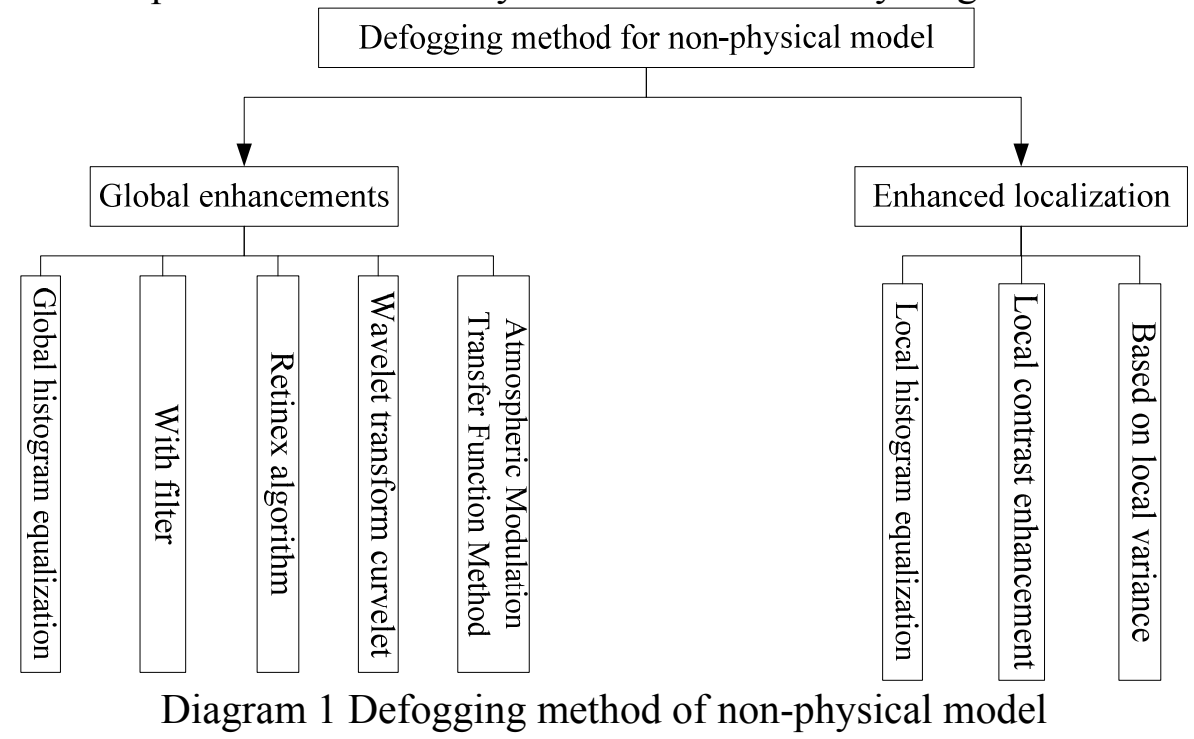

\section{Image enhancement algorithm based on Retinex algorithm}

Under smog condition, the sharpening aim on colorful image is mainly to strengthen contrast ratio of image waiting for procession, broaden brightness and keep its color tone. Therefore, in the spatial image enhancement field, Retinex algorithm has unique advantages for colorful image enhancement. In the description of Retinex theory, object color is mainly depends on 3 wave bands, reflection of object surface is related to nature of object itself, the reflection light of light wave hit onto object is different, which indicates that object color has no direct relations to light source, it can make smog image have clear and bright effect after degradation procession by improving the brightness and darkness degree of RGB color for every pixel. The main methods include global, part, SSR of single scale and multi-scale MSR as well as MSRCR image enhancement algorithm of multi-color recovery, in order to obtain better effect on image procession in smog weather and much more complicated weather, this paper uses MSR as basis and introduces into 3 kinds of different measures according to its characteristic, and then makes comprehensive application to get one kind of clear sharpening algorithm.

The algorithm of combining with global and part is as follows:

(1) Decompose input image $\mathrm{I}(i, j)$ into 3 components image of $\mathrm{I}_{\mathrm{R}}(i, j), \mathrm{I}_{\mathrm{G}}(i, j), \mathrm{I}_{\mathrm{B}}(i, j)$, and then converts pixel gray of every image BYTE type into double type.

(2) Convert it into $\log$-domain $\mathrm{I}^{1}(i, j)=\log I(i, j)$;

(3) Set the initial bit of image $\mathrm{R}(i, j)$ after image enhancement as constant, that is $\mathrm{R}(\mathrm{i} . \mathrm{j})=$ cons $\tan \mathrm{I}$. 
(4) It applies Gaussian filter strength $\mathrm{F}(\mathrm{x}, \mathrm{y})$ to make convolution for the original image, $\mathrm{D}(x, y)=I(x, y) \bullet F(x, y)$ is the image of Gaussian function after weight.

(5) In the log-domain, it uses $\mathrm{G}(x, y)=\mathrm{S}^{1}(x, y)-\log D(x, y)$ expression formula to indicate relevant bright and dark relations of every pixel between pixel point of original image and image after Gaussian weight.

(6) It corrects pixel gray of $\mathrm{R}(i, j)$ through $\mathrm{G}(\mathrm{x}, \mathrm{y})$.

$$
\mathrm{R}^{1}(x, y)=\mathrm{R}(x, y)-G(x, y)
$$

(7) Make linear extension on image after enhancement, finally combine with every component and output it.

When $\mathrm{F}(x, y)$ meets the following formula:

$$
G(x, y)=\sum_{j=1}^{K} W_{\mathrm{i}}\left\{\log \mathrm{I}_{\mathrm{i}}(x, y)-\log \left[I_{i}(x, y) * F(x, y)\right]\right\}
$$

It is regarded as MSR algorithm. contrast.

It adopts this algorithm to make simulation for smog image, and gets the following image
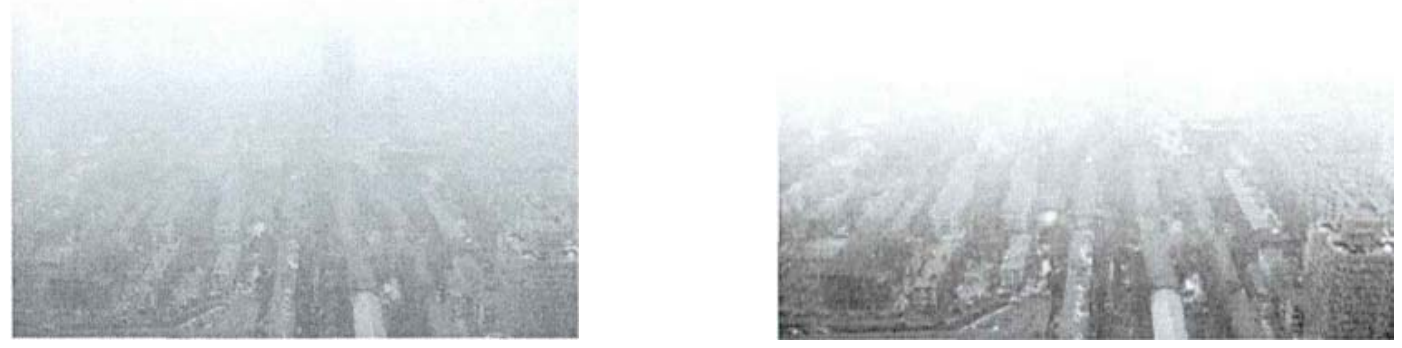

Diagram 2 Comparison result of MSR image by combining with global and part

Such as it is indicated by diagram1, each parameter comparison of smog image, comparison result indicates that brightness of image (b) through original algorithm procession has reduced compared with original image, but its contrast ratio has doubled, and information entropy also increases, while compared with MSR image of combining with global and part, brightness, contrast and information entropy has increased to different extent, and its brightness has greatly enhanced, compared with (b), brightness obviously increases, while contrast ratio and information entropy reduces. Because proper brightness, contrast ratio are favorable for human eyes to make observation, so MSR algorithm of combining with global and part has better articulation through observation by human eyes.

Table 1 Parameter comparison of test 4 of smog image

\begin{tabular}{llll}
\hline & Brightness & Information entropy & Contrast ratio \\
\hline Original image & 157.1950 & 32.1854 & 8.3557 \\
MSR algorithm & 152.6875 & 61.4882 & 9.9451 \\
$\begin{array}{l}\text { MSR of combining } \\
\text { with global and part }\end{array}$ & 174.9766 & 61.3826 & 9.3619 \\
\hline
\end{tabular}

In MSR, it adopts method of multi-scale weighted mean to make procession, but when output image is converted into indicator for indication, its pixel value has negative and exceeds indication range, at this time, it usually needs gain compensation to make adjustment on pixel range of image so that it can get better indication effect.

The expression formula is as follows by adding gain compensation:

$$
\mathrm{R}^{1}{ }_{\mathrm{M}}(x, y)=G * \mathrm{R}_{\mathrm{M}}(x, y)+b
$$

Of which, $\mathrm{G}, \mathrm{b}$ is respectively gain coefficient and compensation coefficient, it usually adopts linear extension to automatically make gain compensation, the calculation process is indicated as follows:

$$
\mathrm{R}^{1}{ }_{\mathrm{M}}(x, y)=\frac{\mathrm{R}_{M_{t}}-R_{\text {min }}}{R_{\text {max }}-R_{\text {min }}} \times d_{\text {max }}
$$


Of which, $\mathrm{R}^{1}{ }_{M}(x, y)$ indicates the output image after algorithm procession, $\mathrm{R}_{\mathrm{M}}(x, y)$ is $\mathrm{MSR}$ image of the i color channel, $R_{\max }, R_{\min }$ are respectively the maximum and minimum of input image pixel, $d_{\max }$ indicates the dynamic range of output equipment.

As for the output value of Retinex, output value in area with high contrast ratio will become bigger or smaller, so it is distributed at both ends of codomain, out put value of detail $\mathrm{K}$ is moderate, so it corresponds to the middle area; this kind of gain function can be indicated as follows:

$$
g_{n_{i}}(x, y)=\left[k_{1} \bullet \frac{2}{b} \bullet N R_{n_{i}} \bullet \exp \left(-k_{2} \bullet N R_{n^{i}}{ }^{2}(x, y) / b\right)+1\right]^{1-\delta_{n}}
$$

In the formula, $\mathrm{k}_{1}, \mathrm{k} 2$, $\mathrm{b}$ all adjusts the constant of gain curve and they will affect gain peak, of which, $\mathrm{k}_{1}, \mathrm{k} 2$, $\mathrm{b}$ will affect the appearance position of gain peak.
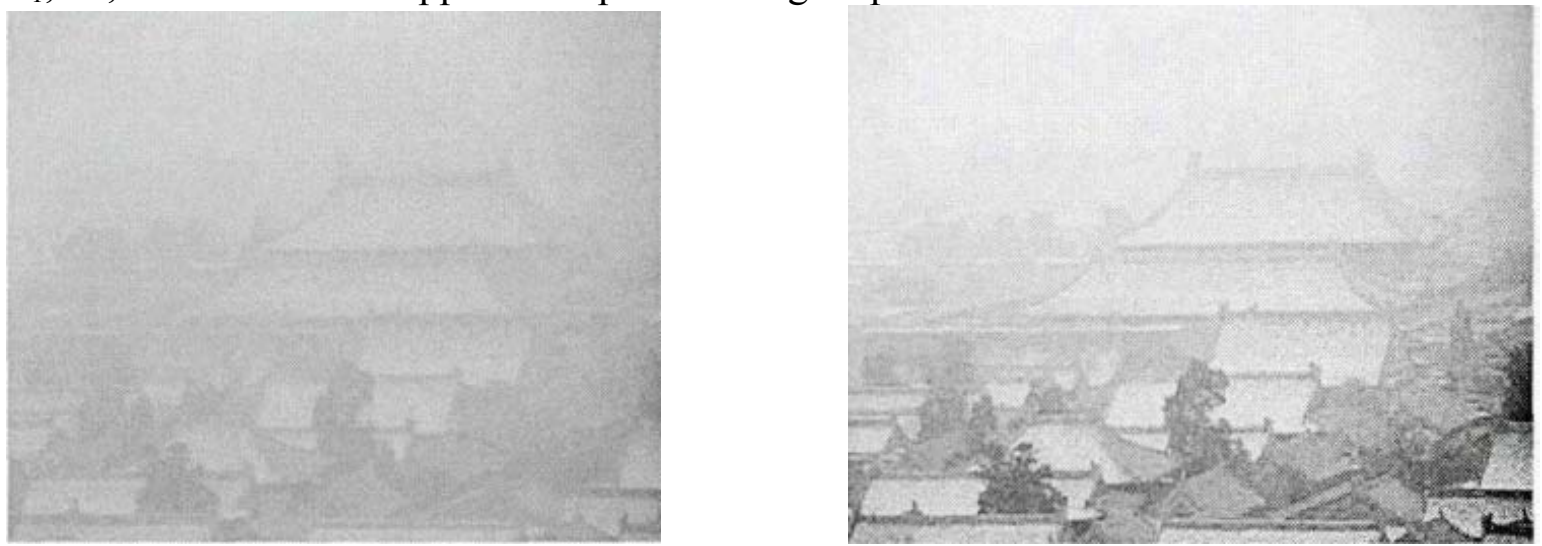

Diagram 3 Comparison chart of MSR procession effect through gain compensation

It makes quantitative analysis on brightness, contrast ratio, information entropy etc of image, it is indicated by table 2 .

\begin{tabular}{llll}
\hline & brightness & information entropy & Contrast ratio \\
\hline original image & 170.2971 & 13.3040 & 7.0650 \\
MSR algorithm & 183.5138 & 43.1590 & 9.4297 \\
MSR of combining & 194.2372 & 38.1707 & 8.9924 \\
$\begin{array}{l}\text { with global and part } \\
\text { Combine with }\end{array}$ & 193.8574 & 38.9944 & 11.7479 \\
$\begin{array}{l}\text { MSR+gain } \\
\text { compensation }\end{array}$ & & & \\
\hline
\end{tabular}

The image brightness of global and part combining with MSR procession has increased, but contrast ratio and information entropy all have slightly reduced, this is due to make up for the background vagueness caused by excessive increase of contrast ratio in the image, the image brightness has slightly reduced by combining with MSR + gain compensation, contrast ratio has slight increase, information entropy has larger increase, value has greatly increased, therefore, it can reflect the indicated gain compensation, it has larger advantage for detail enhancement of each image domain.

The whole algorithm process is as follows:

1. Decompose the input image into 3 gray component images of $\mathrm{R}, \mathrm{G}$, and $\mathrm{B}$.

2. Respectively convert pixel gray of 3 pieces of component image from BYTE type into double type.

3. Use global and part combination, respectively make enhancement procession on 3 pieces of images through MSR algorithm after gain compensation.

4. Compose 3 component images of R, G, B, and then converts it into HIS space and decomposed as 3 component images of $\mathrm{H}, \mathrm{S}, \mathrm{I}$.

5. After procession, integrate 3 pieces of component images into one color image with 24 bytes and finally output this image. 


\section{Smog image sharpening procession based on quality evaluation and feedback}

The main calculation process of this method is as follows: by extracting 3 key visual parameters such as information entropy, contrast ratio and brightness from image, and combine with characteristics of human eyes to redefine these 3 parameters, and then make integration and analysis, the average gray of the overall image can be indicated as follows:

$$
\mathrm{AG}=\frac{1}{M \times N} \sum_{y=0}^{N-1} \sum_{x=0}^{M-1} G(x, y)
$$

Introduce comprehensive evaluation function $\mathrm{CAF}$ :

$$
\mathrm{CAF}=\operatorname{InEn}_{c}^{\alpha} \times A C_{c}^{\beta} \times N G D^{\gamma}
$$

Through plenty of tests, it determines that $\alpha=\beta=\gamma=1$ in the formula is regarded as normalization gray difference, it is indicaed as follows:

$$
N G D=\frac{A O G--\operatorname{dist}(G(x, y)-A O G)}{A O G}
$$

Of which, dist(.) indicates distance operator, AOG indicates the best evaluation gray experienced by human vision, here it takes 127.5, this is the result by theory induction for the most proper gray value. It demonstrates through plenty of tests that the higher of gray value, the higher of image articulation, which means the quality is better.

In CAF evaluation standard, the most important item is gray, when it is closet to standard value of 127.5 , the image quality is the best. Usually effect of smog image after certain articulation algorithm is not the best, while it usually indicates that gray value has concentrated distribution, from introduction of the above paper we can know that equalization of histogram is relatively better as for the balance effect of image gray. In the space of colorful image, HIS space can be separately operated out of sensitivity and this space is most approximate $t$ visual characteristics of human and uses CAF as evaluation standard, establishes new image sharpening algorithm and realizes output of optimal image. The detailed algorithm process is indicated by the following diagram 4:

1. It makes ft evaluation based on CAF standard for the original image and records CAF value.

2. It makes sharpening procession algorithm procession for the image in this paper.

3.It makes quality evaluation of CAF standard for the output image of algorithm, and records every data, makes comparison for original value of CAF: if it is less than original value, then directly inputs this image and FLiij, which indicates effect of this sharpening algorithm is not good, and needs further improvement, if it is bigger than original value, makes histogram equalization for image, and then continue to make cycle procession until the tested CAF value is not bigger than value of the prior image, then directly outputs this image and it is the optimal image.

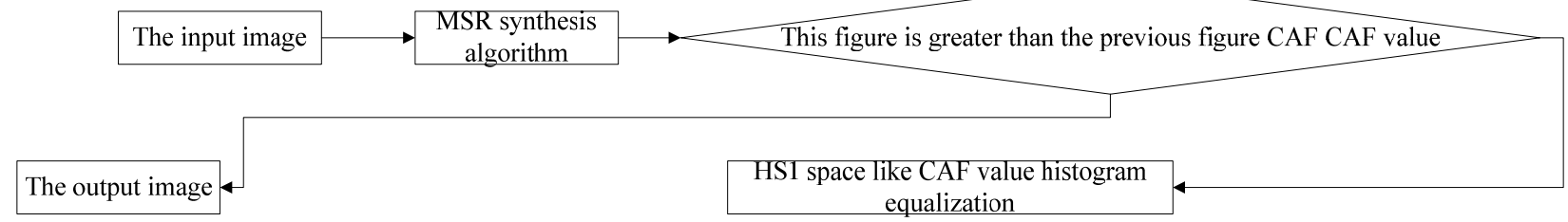

Diagram 4 The algorithm process chart based on quality evaluation and feedback

\section{Demonstration on algorithm test}

Test condition: take photograph from viaduct on city road, time: 7:20 in the morning, weather: fuggy, wind grade: 0, humidity: $20 \%$, outdoor temperature: $180 \mathrm{C}$, distance range $100 \mathrm{~m}-300 \mathrm{~m}$. Equipment choice: one set of digital camera (core element CCD), one set of computer, Matlab(2012b) procession software.

As for test image: the smog image in Beijing(diagram 5), carries out sharpening procession in this paper and gets clear image, uses quality evaluation and feedback system designed in this chapter to make further sharpening, test data and record are indicated by table 3 . 
Table 3 Quality evaluation and feedback data of smog image

\begin{tabular}{llll}
\hline & Original image & algorithm of this paper & Feedback condition \\
\hline CAF & 21.9002 & 43.8030 & 43.8343 \\
\hline
\end{tabular}

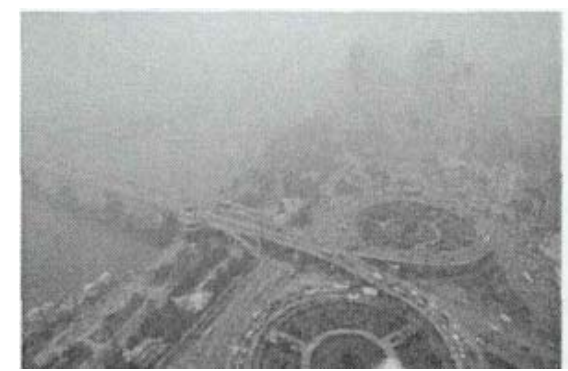

(a) Original image

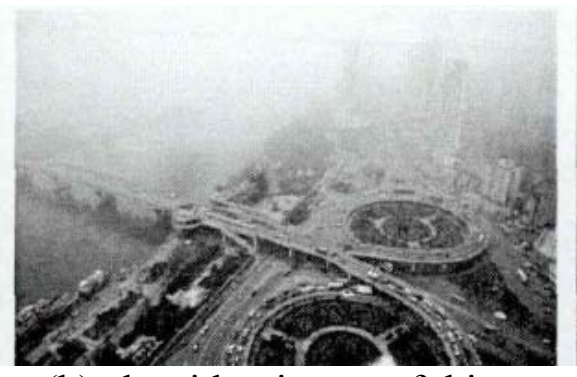

(b) algorithm image of this paper

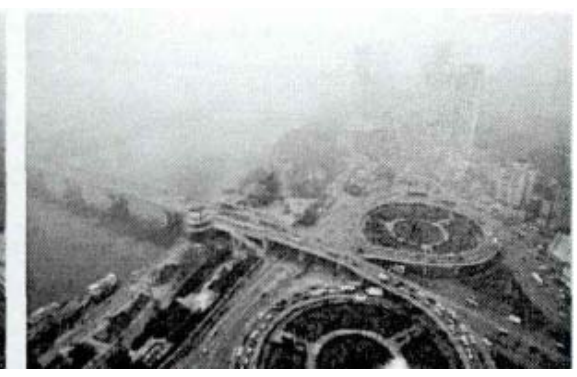

(c) optimal image

Diagram 5 Quality and evaluation feedback chart on smog image in Beijing

The color tone of smog image is yellow and belongs to condition with serious smog, the overall contrast degree is low, it is indicate by the above diagram after sharpening algorithm procession in this paper, the contrast degree of CAF standard in image increase by nearly 2 times and CAF value increases from 21.9002 to 43.8343 , the enhancement effect is obvious. It makes quality feedback on image for 2 times, we can find that the contrast ratio in CAF standard reaches convergence and maintains at 43.8343, at this time the output optimal image is indicated by diagram 5.3.2 $\mathrm{C}$. The average gray of diagram (b) and diagram(c) all relatively approaches to standard value 127.5, and diagram (b) has little error with standard error, CAF value has little difference compared with (c). Therefore, it can be demonstrated again that algorithm in this paper has obvious advantages for sharpening of smog image, and this quality evaluation and feedback system has better effect on further sharpening of image.

\section{Summary}

In this paper, sharpening procession of image under smog weather condition is the importamt problmes concerned in the field of digital image procession, applying digital image procession method can effectively restore scene information. This paper has stronger practicability and its research progress has great application values in fields such as road and traffic monitoring, remote detection, target identification and military field., This paper starts from analysis of Retinex algorithm, and makes simulation and comparison on algorithms such as global, SSR, MSR, MSRCR, it summarizes the advantages of MSR. Then it makes overall and part combination as well as improvment in gain compensation, and uses histogram equalization as subsequent procession, it alsk makes comparison on several kinds of algorithms on 2 pieces of images from aspects objective and subjective image quakity evaluation, which demonstartes that algorithm in this paper has better sharpening effect.

\section{References}

[1] Ruan Qiuqi. Digital Image Treatment. Beijing: Publishing House of Electronics Industry, 2007. p71-73.

[2] Zhang Xinlong, Wang Ronggui and Zhang Xuan etc. Enhancement Calculation and Algorithm of

Smog Image.Journal of Chinese Graphics, Vol 16(8), 2011,p 1359-1368..

[3] Ma Yunfei, He Wenzhang. Enhancement Method of Smog Image Based on Small Wave

Transformation [J].Computer Application and Software, Vol 28(2), 2011,p

$71-72,95$.

[4] Lin Ying. Defogging Image Procession of Homomorphic Filtration Based on Small Wave

Transformation [J]. Journal of Longyan University, vol 26(6)， 2009， p32-36.. 
[5] Huang Yiming. Ereserch on Image Enhancement Algorithm under Smog Weather.Dalian: Dalian University of Technology, 2013

[6] Guo Fan, Cai Zixing, Xie Bin etc. Overview and Expectation of Image Defogging Technology. Computer Application, Vol 30(9), 2010, p 2417-2421.

[7] Zhang Xinlong, Wang Ronggui, Zhang Xuan etc. Enhancement Model and Algorithm of Smog Image. Journal of Chinese Graphics, Vol 16(8),2011, Vol 1359-1368. 